\title{
Effect of Oxygen Flow Rate on the Optical, Electrical, and Mechanical Properties of DC Sputtering ITO Thin Films
}

\author{
Chuen-Lin Tien $\mathbb{D}^{1,2}$ Hong-Yi Lin, ${ }^{2}$ Chih-Kai Chang, ${ }^{2}$ and Chien-Jen Tang ${ }^{3}$ \\ ${ }^{1}$ Department of Electrical Engineering, Feng Chia University, Taichung 40724, Taiwan \\ ${ }^{2} \mathrm{Ph}$. D. Program of Electrical and Communications Engineering, Feng Chia University, Taichung 40724, Taiwan \\ ${ }^{3}$ Department of Photonics, Feng Chia University, Taichung 40724, Taiwan
}

Correspondence should be addressed to Chuen-Lin Tien; cltien@fcu.edu.tw

Received 17 November 2017; Accepted 9 January 2018; Published 7 February 2018

Academic Editor: Shuan-Yu Huang

Copyright (C) 2018 Chuen-Lin Tien et al. This is an open access article distributed under the Creative Commons Attribution License, which permits unrestricted use, distribution, and reproduction in any medium, provided the original work is properly cited.

\begin{abstract}
This study presents the effect of oxygen flow rate on the optical, electrical, and mechanical properties of indium tin oxide (ITO) thin films prepared by the DC magnetron sputtering technique. The oxygen flow rate was varied from 10 to $50 \mathrm{sccm}$. The ITO thin films deposition under different oxygen flow rates exhibits different properties. We used an optical spectrometer to measure the optical transmittance and a four-point probe instrument to determine the resistivity. A home-made Twyman-Green interferometer was used to evaluate residual stress and a microscopic interferometer was used to measure the surface roughness of ITO thin films. The experimental results show that the average optical transmittance is larger than $85 \%$ in visible range; the electrical resistivity has a minimum $6.85 \times 10^{-4} \mathrm{ohm}-\mathrm{cm}$ for the oxygen flow of $10 \mathrm{sccm}$. The residual stress is varied from $-0.15 \mathrm{GPa}$ to $-0.34 \mathrm{GPa}$ in the range of $10-50 \mathrm{sccm}$. The root-mean-square ( $\mathrm{rms}$ ) surface roughness is changed from $2.64 \mathrm{~nm}$ to $2.74 \mathrm{~nm}$ as the oxygen flow rate increases. The results show that the oxygen flow rate has significant influence on the electrical resistivity, residual stress, and surface roughness of the ITO thin film.
\end{abstract}

\section{Introduction}

The transparent conductive oxide films (TCO) had excellent physical and chemical properties, and the coating process parameters have significant influence on the optical, electrical, and structural properties and residual stress of the transparent conductive oxide films. Indium tin oxide (ITO) thin film is one kind of transparent conductive films. Due to its high transmittance and good physical and chemical properties [1,2], it is widely used in optical devices like flat panel displays, solar cells, sensors, and so on [3-5]. There are many different methods for the fabrication of ITO thin films including the magnetron sputtering method $[6,7]$, thermal evaporation method [8], plasma ion-assisted evaporation [9], reactive electron-beam evaporation [10], and sol-gel method [11]. It is well known that almost all thin films, produced by the various deposition techniques, have residual stresses. Residual stress that develops during thin film growth is critical issue for many applications. Films stress causes many undesirable phenomena, for example, the cracking or peeling of the film and the bending of the substrate. The precise measurement of residual stress in thin films is helpful to understand the stress behaviour and related effects. DC magnetron sputter deposition has generally been used in industrial production lines for the deposition of ITO films because this method yields homogeneous ITO films with low resistivity and good reproducibility. Therefore, in order to improve the quality of the ITO thin films, the surface characteristics, residual stresses, and optical and electrical properties of DC magnetron sputtering ITO thin films have been investigated experimentally.

There are many papers to explore the optical, electrical, and structural properties of ITO thin films prepared by different deposition methods. However, there are few studies to report the effect of oxygen flow rate on the residual stress and surface roughness of sputtering ITO thin films [12-14]. This study investigated the effect of oxygen flow rate on optical, mechanical, and electrical properties and the surface roughness of ITO thin films prepared by DC magnetron sputtering technique. The optical transmittance spectra of ITO films 
were scanned by a UV-Visible spectrometer. The resistivity of these films was measured by a four-point probe instrument. The residual stress was evaluated by a home-made Twyman-Green interferometer. The surface roughness was determined by a Linnik microscopic interferometer.

\section{Experimental Methods}

2.1. DC Magnetron Sputtering. ITO thin films were deposited using DC magnetron reactive sputtering. The vacuum system consisted of a turbo molecular pump backed by a mechanical pump. A three-inch disk of In-10 wt\% Sn alloy was used as a sputtering target. The spacing of the target to substrates was $80 \mathrm{~mm}$. Before sputtering, the target was presputtered for about 10 min with a shutter covering the target in order to remove the adsorbed gas on the surface of the ITO target. The working gas consisted of a mixed gas of $\mathrm{Ar}$ and $\mathrm{O}_{2}$, the flow rates of which were independently controlled by mass flow controllers. A DC discharge was sustained by a power supply operated under the constant-current mode with $150 \mathrm{~mA}$. The constant-current mode can reduce spikes and arcing; thus plasma conditions are more stable in coating process. The base pressure of the system was $2.7 \times 10^{-4} \mathrm{~Pa}$. To minimize the oxidation of the target, $\mathrm{Ar}$ and $\mathrm{O}_{2}$ were introduced near the target and the substrate, respectively. The flow rates of oxygen reactive gas were varied from 10 to $50 \mathrm{sccm}$. Both BK7 glass and silicon wafer were employed as coating substrates, where the former was used for measuring the transmittance and residual stress of ITO thin films and the latter for measuring the other properties. The coating substrates were not heated and were assumed to be at near room temperature during the film deposition.

2.2. Optical Properties. Analysis of the optical properties of deposited films was carried out. The relative transmittance spectra of ITO thin films deposited at different oxygen flow rates were measured. In this work, the UV-Visible spectrometer (Chrom Tech., CT-2200) was used to measure the transmission spectrum from $350 \mathrm{~nm}$ to $850 \mathrm{~nm}$. The envelope method was used to determine three optical constants, namely, the refractive index $(n)$, extinction coefficient $(k)$, and physical thickness $(d)$. These were determined from the transmittance spectrum with loss absorption [15].

2.3. Residual Stress Measurements. Stress in thin films is primarily composed of a thermal stress and an intrinsic stress [16]. The thermal stress is due to the difference in the thermal expansion coefficients between the coating and substrate materials. The intrinsic stress is induced during the deposition process, but the mechanisms are complex and not yet fully understood. To study thin film stress issues, it is necessary to understand the stress mechanisms and their effects. Therefore, the precise measurement of stress in thin films is of considerable importance in numerous industrial applications.

We used a home-made Twyman-Green interferometer associated with the fast Fourier transform (FFT) method to measure the radius of curvature of substrate before and after

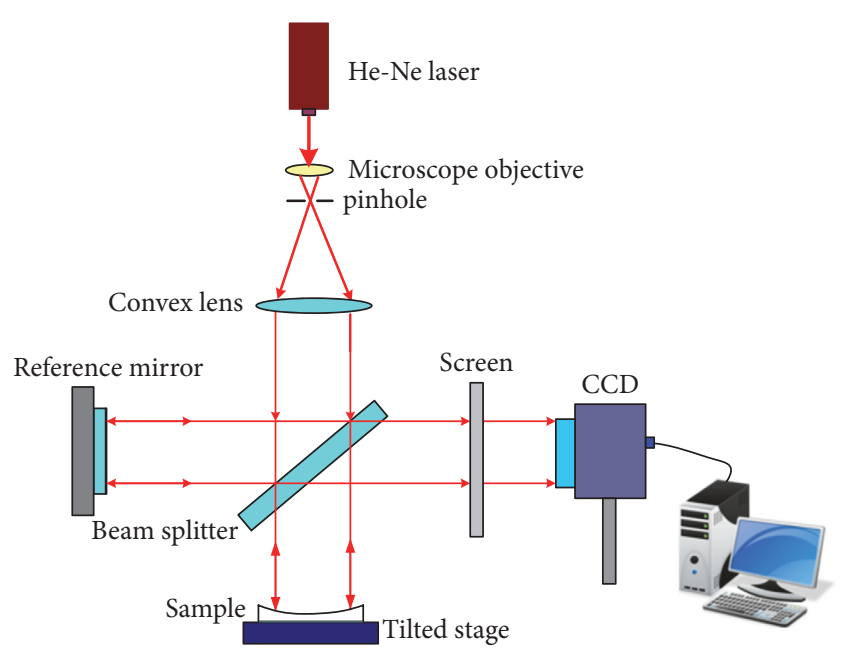

FIGURE 1: Stress measuring instrument based on Twyman-Green interferometer.

thin film deposition $[17,18]$. The substrate was a circular BK7 glass with a diameter of $25.4 \mathrm{~mm}$ and thickness $t_{s}=1.5 \mathrm{~mm}$. The schematic diagram of the stress measuring apparatus is shown in Figure 1. The laser beam passed through a spatial filter and then through a lens to form a plane wave front. The plane wave front was divided into different amplitudes by a beam splitter. The reflected beam and the transmitted beam traveled to a reference plate and a tested sample. The two beams were recombined and traveled toward a CCD camera after being reflected by both the reference plate and the tested sample. A digital CCD camera was used to record the interference fringe and the stress in the thin films was measured by the fast Fourier transform (FFT) method [18]. A thin film's surface deformation can be readily obtained by subtraction of the surface contours before and after film deposition. The residual stress of the thin films can be determined by the modified Stoney equation [19]:

$$
\sigma=\frac{1}{6} \frac{E_{s}}{1-v_{s}} \frac{t_{s}^{2}}{t_{f}}\left(\frac{1}{R}-\frac{1}{R_{o}}\right),
$$

where $\sigma$ is residual stress of the films; $E_{s}$ is Young's modulus $\left(E_{s}=81 \mathrm{GPa}\right)$ of the BK7 substrate; $\nu_{s}$ is Poisson's ratio $\left(\nu_{s}=\right.$ 0.208 ) of the BK7 substrate; $R_{0}$ is the radius of curvature of the $\mathrm{BK} 7$ substrate before coating; $R$ is the radius of curvature of the BK7 substrate after coating; $t_{s}$ is the thickness of the BK7 substrate; $t_{f}$ is the thickness of the films. By convention, $\sigma$ is negative for compressive stress and positive for tensile stress. Tensile stress is present in the film if the curvature of the substrate is concave; conversely, the stress is compressive if the curvature of the substrate is convex.

2.4. Surface Roughness Measurements. The surface morphology and microstructure of the thin films depended on the properties of the films and fabrication parameters. There are many ways to measure the roughness of the films. We usually use root-mean-square (rms) roughness to evaluate the surface roughness of the thin films. We used a home-made 


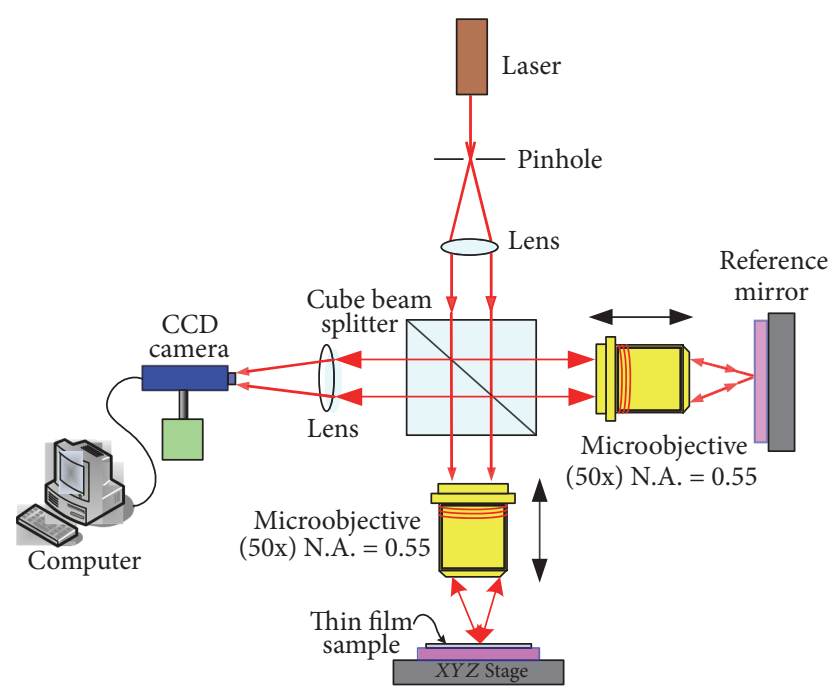

FIGURE 2: Measurement of surface roughness by using Linnik microscopic interferometer.

Linnik microscopic interferometer to evaluate the surface roughness of thin films [20]. The Linnik microscopic interferometer was combined with the fast Fourier transform method to build up a surface roughness measurement system, as shown in Figure 2. The interference patterns were captured by a CCD camera. The fast Fourier transform (FFT) technique was used for the phase extraction. A phase unwrapping algorithm is needed to remove the discontinuities by adding or subtracting multiples of $2 \pi$ to a pixel until the difference between it and its adjacent pixel is less than $\pi$. A Gaussian filter is used to separate the roughness profile from the original profile. The mean surface profile is then subtracted from the surface height profile to obtain the roughness profile. The root-mean-square value of surface roughness is determined by the numerical analysis with MATLAB program.

\section{Results and Discussion}

The optical transmittance of the films was measured by a UV-Visible spectrophotometer in the wavelength range $350-750 \mathrm{~nm}$. Figure 3 shows transmittance spectra of the ITO thin films deposited under different oxygen flow rates. The transmittance of the ITO thin films is not a function of the oxygen flow rate, but all samples are over $85 \%$ in the visible region. At oxygen flow rate of $30 \mathrm{sccm}$, the transmittance of the ITO thin films has a maximum value of $88.7 \%$ for the wavelength of $500 \mathrm{~nm}$. The spectra of the ITO films were obtained by scanning from $350 \mathrm{~nm}$ to $850 \mathrm{~nm}$. The thickness of ITO films in various conditions was determined by transmittance spectrum. The average refractive index $(n)$ and the extinction coefficient $(k)$ in visible range vary in range of 2.01-2.04 and $5.6 \times 10^{-3}-6.5 \times 10^{-3}$, respectively. However, no obvious correlation between $n$ and $k$ is observed. Figure 4 shows the ITO film thickness as a function of the oxygen flow rate.

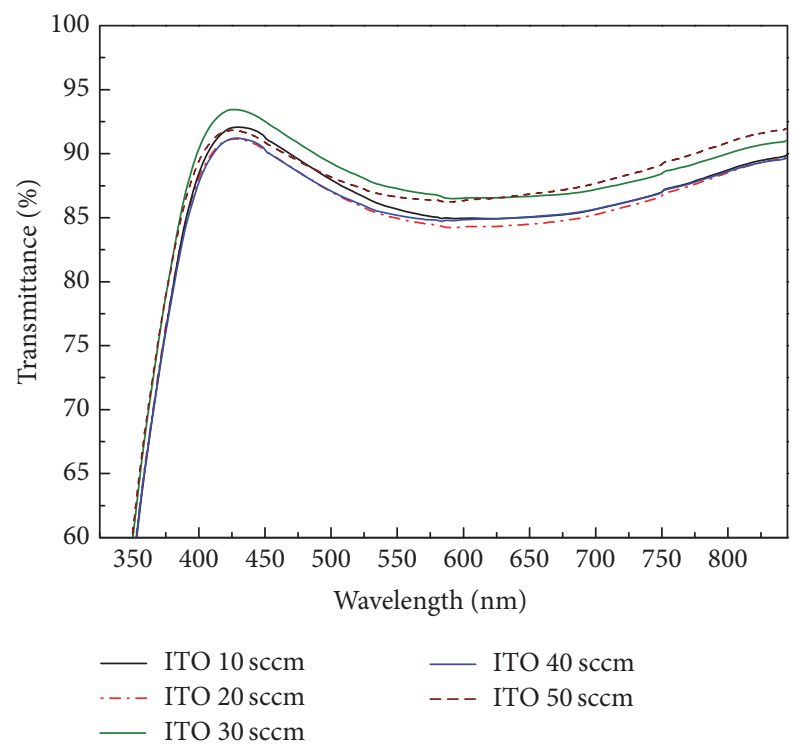

FIGURE 3: Transmittance spectra of ITO thin films deposited under different oxygen flow rates.

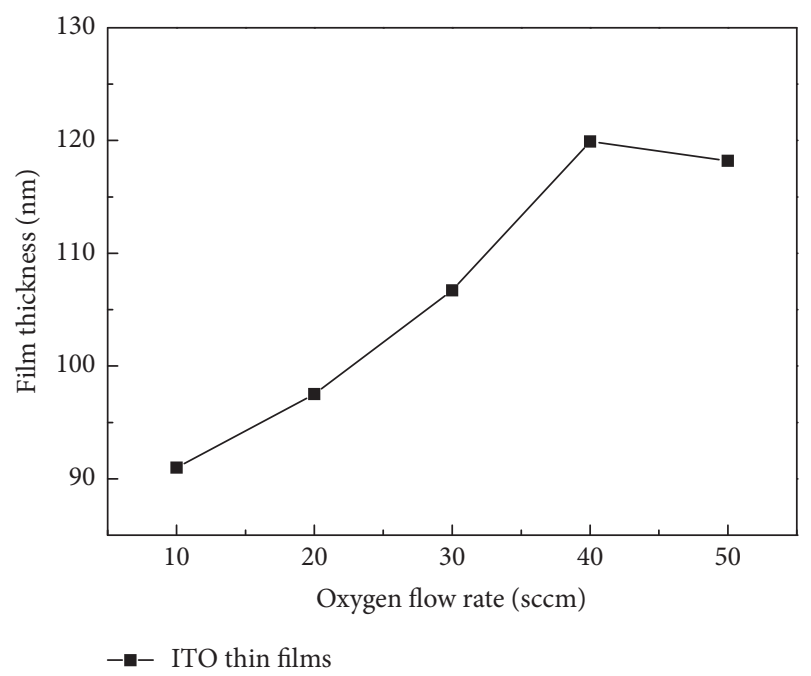

FIGURE 4: Film thickness as a function of oxygen flow rate.

In this study, a sheet resistance meter with four-point probe was used to measure the sheet resistance and resistivity of thin films. Figure 5 shows the resistivity and sheet resistance as a function of the oxygen flow rate. The resistivity and sheet resistance increase with increasing the oxygen flow rate from 10 to $30 \mathrm{sccm}$, and then both decrease as the oxygen flow rate is larger than $30 \mathrm{sccm}$. In the film deposition experiments, the oxygen flow rate was $10 \mathrm{sccm}$ and the working pressure was maintained at $6.7 \times 10^{-4} \mathrm{~Pa}$; ITO thin films exhibit a lower resistivity and sheet resistance. A maximum resistivity of $1.56 \times 10^{-3} \Omega-\mathrm{cm}$ is found at the oxygen flow rate of $30 \mathrm{sccm}$. The lowest resistivity of $6.85 \times 10^{-4} \Omega$-cm and a minimum sheet resistance of $75.8 \Omega / \mathrm{sq}$. were achieved at the oxygen flow rate of $10 \mathrm{sccm}$. This low resistivity and sheet resistance may be attributed to a formation of $\mathrm{SnO}_{2}$ 


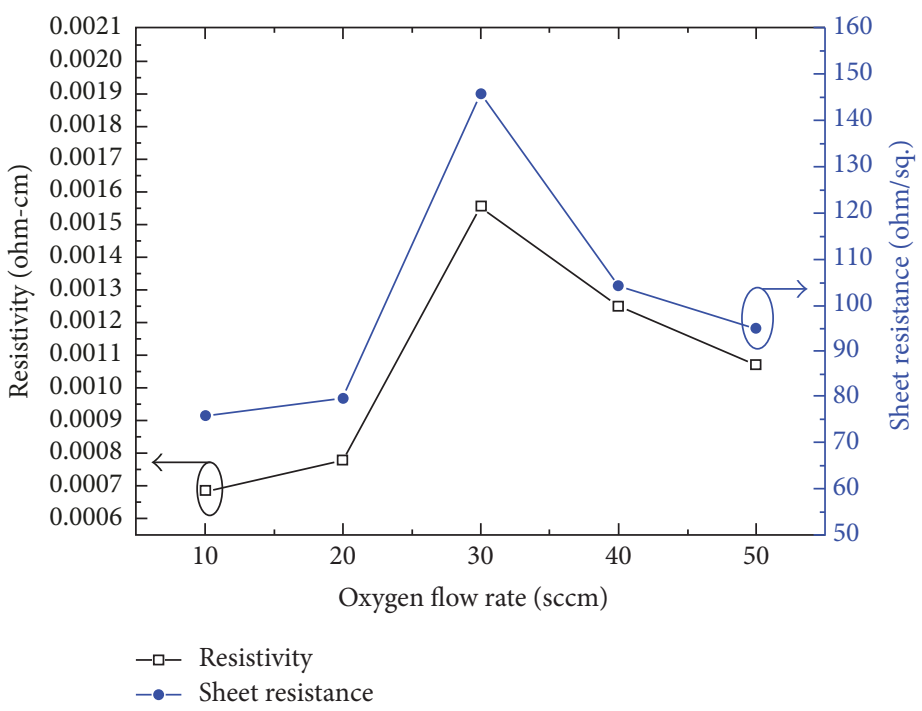

FIGURE 5: Resistivity and sheet resistance of ITO thin films deposited under different oxygen flow rates.

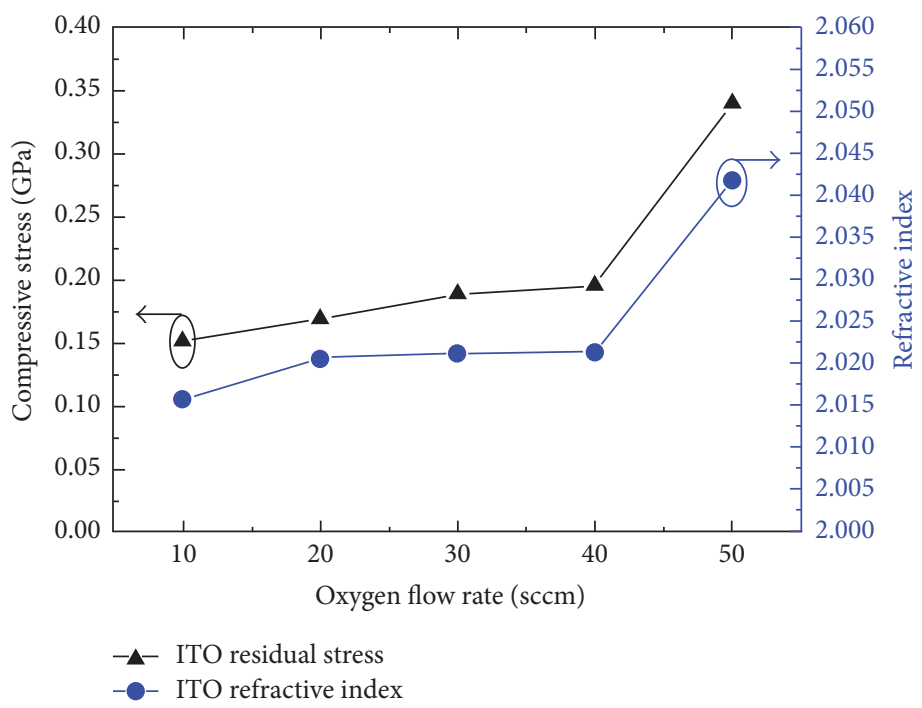

FIGURE 6: Residual stress and refractive index of ITO thin films as a function of oxygen flow rate.

in the films resulting in high carrier mobility. The electrical resistivity of the ITO films is considered to be sensitive to the oxygen flow rate in the mixture of the discharge gas (argon) and reactive gas (oxygen). At oxygen flow rates from 10 to $30 \mathrm{sccm}$, the increase in resistivity and sheet resistance was due to gradual decrease of the carrier mobility. The mobility was slightly enhanced with increasing oxygen flow rates from 30 to $50 \mathrm{sccm}$. It is well known for the ITO films that the electrons are released from the substitutional entered $\mathrm{Sn}$ atoms in the sublattice and from the doubly charged oxygen vacancies. Tin can exist as either $\mathrm{SnO}$ or $\mathrm{SnO}_{2}$. The presence of $\mathrm{SnO}_{2}$ would result in $n$ doping of the lattice because the dopant would add electrons to the conduction band [21]. Furthermore, many studies have reported that the resistivity of ITO thin films is strongly dependent on the oxidation state during film deposition [22-25]. As pointed out above, the charge carriers of the ITO thin films are either contributed by $\mathrm{Sn}^{+4}$ ion or oxygen vacancies. The incorporation of oxygen atoms into the ITO films causes the decrease of oxygen vacancies and then gives rise to higher resistivity of ITO films [26]. When the oxygen vacancies in ITO thin films are fully filled, it leads to thin films growing more dense. This cannot only decrease the defects, but slightly improve the mobility in ITO films [27].

Film stress is an important factor in the adhesion and stability of thin films. Figure 6 shows the residual compressive stress and refractive index as a function of the oxygen flow rate. Both values increase as the oxygen flow rate increases. The residual stress in all ITO thin films is a compressive stress with values ranging from $-0.15 \mathrm{GPa}$ to $-0.34 \mathrm{GPa}$. In this work, the plasma bombardment energy increases as oxygen flow rate increases during thin film deposition; the 


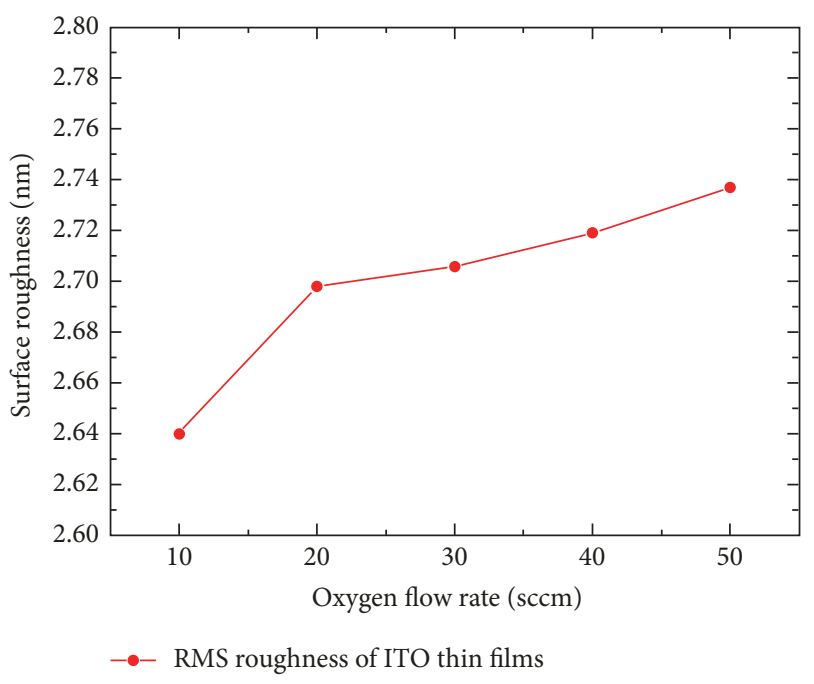

FIGURE 7: Surface roughness of ITO thin films as a function of oxygen flow rate.

ITO thin film growth becomes more dense (i.e., refractive index increasing) to form a compressive stress. Mattox [28] reported that energetic particle bombardment can introduce a compressive film stress. In a low-pressure sputtering deposition, energetic particle bombardment may induce high compressive film stress due to the recoil implantation of surface atoms. Besides, Windischmann [29] suggested that the compressive stress in sputtered films appears to be momentum rather than energy driven. However, no one model can explain all experimental data; the atomic peening model [30-32] appears to be more appropriate for sputtering deposition involving energy particle bombardment of the growing films leading to compressive stresses.

For the surface roughness measurements, the roughness can be characterized by several parameters and functions. The average roughness $\left(R_{a}\right)$ is the most widely used because it is a simple parameter to obtain when compared to others. The root mean square (RMS) of roughness $(R q)$ is a function that takes the square of the measures. The surface roughness at a certain area is determined by the height differences of all the distinct points at this area. RMS roughness is the mean of the root for the deviation from the standard surface to the indicated surface. The measurement results show that the RMS surface roughness of ITO films increases with increasing of the oxygen flow rate, as shown in Figure 7. The RMS surface roughness is changed from $2.64 \mathrm{~nm}$ to $2.74 \mathrm{~nm}$ in the range of $10-50 \mathrm{sccm}$. It is clear that the ITO film prepared with low oxygen flow rate had a low RMS roughness value $(2.64 \mathrm{~nm})$ than that of the ITO films prepared in the high oxygen flow rate under the same deposition conditions. The reason may be due to bombardment of the more energetic particles on the growing film which causes the rough surface. We also found that the RMS surface roughness of ITO thin films increased with a corresponding increase in film thickness (Figure 4), while a relatively small surface roughness was obtained for the ITO films that were deposited at oxygen flow rate of $10 \mathrm{sccm}$. The lower flow rate helps in producing a smooth ITO film with suitable opto-electrical properties on substrates without heating during deposition and/or additional post-annealing treatments.

\section{Conclusions}

This work demonstrates the influence of the oxygen flow rate on optical, electrical, and mechanical properties and surface roughness of DC sputtering ITO thin films. The ITO thin films were deposited on glass substrates and silicon wafer by DC magnetron sputtering technique under different oxygen flow rates. The ITO films prepared under the optimal deposition conditions had an optical transmittance of $85 \%$ in the visible spectrum. The experimental results show that ITO thin films are not a function of the oxygen flow rate, but optical transmittance of all ITO films is over $85 \%$ in the visible region. At oxygen flow rate of $30 \mathrm{sccm}$, the transmittance of the ITO thin film has a maximum value of $88.7 \%$ for the wavelength of $500 \mathrm{~nm}$. The electrical resistivity measurements reveal that the sheet resistance values of the ITO thin films increase with the increasing oxygen flow rate, and the electrical resistivity has a minimum of $6.85 \times$ $10^{-4} \mathrm{ohm}-\mathrm{cm}$ for the oxygen flow rate of $10 \mathrm{sccm}$. The residual stress and surface roughness increase as the oxygen flow rate increases in the range of $10-50 \mathrm{sccm}$. In this study, the optimum depositing parameter is the oxygen flow rate of $10 \mathrm{sccm}$ which results in a high transmission of $88 \%$, the lowest resistivity of $6.85 \times 10^{-4} \mathrm{ohm}-\mathrm{cm}$, a lower residual stress of $-0.15 \mathrm{GPa}$, and the RMS surface roughness of $2.64 \mathrm{~nm}$.

\section{Conflicts of Interest}

The authors declare that they have no conflicts of interest.

\section{Acknowledgments}

This work was supported by the Ministry of Science and Technology (MOST) of Taiwan under Grants MOST 1032221-E-035-032 and MOST 104-2221-C-035-058-MY2. The authors appreciate the Precision Instrument Support Center of Feng Chia University for providing the fabrication and measurement facilities.

\section{References}

[1] M. Bender, W. Seelig, C. Daube, H. Frankenberger, B. Ocker, and J. Stollenwerk, "Dependence of oxygen flow on optical and electrical properties of DC-magnetron sputtered ITO films," Thin Solid Films, vol. 326, no. 1-2, pp. 72-77, 1998.

[2] A. Salehi, "The effects of deposition rate and substrate temperature of ITO thin films on electrical and optical properties," Thin Solid Films, vol. 324, no. 1-2, pp. 214-218, 1998.

[3] D. V. Morgan, Y. H. Aliyu, R. W. Bunce, and A. Salehi, "Annealing effects on opto-electronic properties of sputtered and thermally evaporated indium-tin-oxide films," Thin Solid Films, vol. 312, no. 1-2, pp. 268-272, 1998.

[4] L.-J. Meng and M. P. dos Santos, "Structure effect on electrical properties of ITO films prepared by RF reactive magnetron sputtering," Thin Solid Films, vol. 289, no. 1-2, pp. 65-69, 1996. 
[5] O. J. Gregory, Q. Luo, and E. E. Crisman, "High temperature stability of indium tin oxide thin films," Thin Solid Films, vol. 406, no. 1-2, pp. 286-293, 2002.

[6] K. Zhang, F. Zhu, C. H. A. Huan, and A. T. S. Wee, "Effect of hydrogen partial pressure on optoelectronic properties of indium tin oxide thin films deposited by radio frequency magnetron sputtering method," Journal of Applied Physics, vol. 86, no. 2, pp. 974-980, 1999.

[7] H.-Y. Yeom, N. Popovich, E. Chason, and D. C. Paine, "A study of the effect of process oxygen on stress evolution in d.c. magnetron-deposited tin-doped indium oxide," Thin Solid Films, vol. 411, no. 1, pp. 17-22, 2002.

[8] F. Zhu, C. H. A. Huan, K. Zhang, and A. T. S. Wee, "Investigation of annealing effects on indium tin oxide thin films by electron energy loss spectroscopy," Thin Solid Films, vol. 359, no. 2, pp. 244-250, 2000.

[9] S. Laux, N. Kaiser, A. Zöller, R. Götzelmann, H. Lauth, and H. Bernitzki, "Room-temperature deposition of indium tin oxide thin films with plasma ion-assisted evaporation," Thin Solid Films, vol. 335, no. 1-2, pp. 1-5, 1998.

[10] K. R. Narasimha, "Optical and electrical properties of indiumtin oxide films," Indian Journal of Pure \& Applied Physics, vol. 42, no. 3, pp. 201-204, 2004.

[11] R. Bel Hadj Tahar, T. Ban, Y. Ohya, and Y. Takahashi, "Electronic transport in tin-doped indium oxide thin films prepared by solgel technique," Journal of Applied Physics, vol. 83, no. 4, pp. 21392141, 1998.

[12] L.-J. Meng, J. Gao, R. A. Silva, and S. Song, "Effect of the oxygen flow on the properties of ITO thin films deposited by ion beam assisted deposition (IBAD)," Thin Solid Films, vol. 516, no. 16, pp. 5454-5459, 2008.

[13] Y. J. Kim, S. B. Jin, S. I. Kim, Y. S. Choi, I. S. Choi, and J. G. Han, "Effect of oxygen flow rate on ITO thin films deposited by facing targets sputtering," Thin Solid Films, vol. 518, no. 22, pp. 6241-6244, 2010.

[14] A. Chen, K. Zhu, H. Zhong, Q. Shao, and G. Ge, "A new investigation of oxygen flow influence on ITO thin films by magnetron sputtering," Solar Energy Materials \& Solar Cells, vol. 120, pp. 157-162, 2014.

[15] J. C. Manifacier, J. Gasiot, and J. P. Fillard, "A simple method for the determination of the optical constants $n, k$ and the thickness of a weakly absorbing thin film," Journal of Physics E: Scientific Instruments, vol. 9, no. 11, pp. 1002-1004, 1976.

[16] J. A. Thornton and D. W. Hoffman, "Stress-related effects in thin films,” Thin Solid Films, vol. 171, no. 1, pp. 5-31, 1989.

[17] C.-L. Tien and H.-D. Zeng, "Measuring residual stress of anisotropic thin film by fast Fourier transform," Optics Express, vol. 18, no. 16, pp. 16594-16600, 2010.

[18] C.-L. Tien, K.-C. Yu, T.-Y. Tsai, and M.-C. Liu, "Effect of RF power on the optical, electrical, mechanical and structural properties of sputtering Ga-doped $\mathrm{ZnO}$ thin films," Applied Surface Science, vol. 354, pp. 79-84, 2015.

[19] G. G. Stoney, "The tension of metallic films deposited by electrolysis," Proceedings of the Royal Society A Mathematical, Physical and Engineering Sciences, vol. 82, no. 553, pp. 172-175, 1909.

[20] C.-L. Tien, K.-C. Yu, T.-Y. Tsai, C.-S. Lin, and C.-Y. Li, "Measurement of surface roughness of thin films by a hybrid interference microscope with different phase algorithms," Applied Optics, vol. 53, no. 29, pp. H213-H219, 2014.
[21] L.-J. Meng, A. Maçarico, and R. Martins, "Study of annealed indium tin oxide films prepared by rf reactive magnetron sputtering," Vacuum, vol. 46, no. 7, pp. 673-680, 1995.

[22] H. Kim, C. M. Gilmore, A. Piqué et al., "Electrical, optical, and structural properties of indium-tin-oxide thin films for organic light-emitting devices," Journal of Applied Physics, vol. 86, no. 11, pp. 6451-6461, 1999.

[23] F. Kurdesau, G. Khripunov, A. F. da Cunha, M. Kaelin, and A. N. Tiwari, "Comparative study of ITO layers deposited by DC and RF magnetron sputtering at room temperature," Journal of NonCrystalline Solids, vol. 352, no. 9-20, pp. 1466-1470, 2006.

[24] V. Teixeira, H. N. Cui, L. J. Meng, E. Fortunato, and R. Martins, "Amorphous ITO thin films prepared by DC sputtering for electrochromic applications," Thin Solid Films, vol. 420-421, pp. 70-75, 2002.

[25] K.-S. Tseng and Y.-L. Lo, "Effects of cumulative ion bombardment on ITO films deposited on PET and Si substrates by DC magnetron sputtering," Optical Materials Express , vol. 4, no. 4, pp. 764-775, 2014.

[26] T. C. Gorjanc, D. Leong, C. Py, and D. Roth, "Room temperature deposition of ITO using R.F. Magnetron sputtering," Thin Solid Films, vol. 413, no. 1-2, pp. 181-185, 2002.

[27] J.-H. Gu, J.-L. Si, J.-X. Wang, Y.-Y. Feng, X.-Y. Gao, and J.-X. Lu, "Indium-tin oxide films obtained by DC magnetron sputtering for improved Si heterojunction solar cell applications," Chinese Physics B, vol. 24, no. 11, Article ID 117703, 2015.

[28] D. M. Mattox, "Particle bombardment effects on thin-film deposition: A review," Journal of Vacuum Science \& Technology A, vol. 7, no. 3, pp. 1105-1114, 1989.

[29] H. Windischmann, "Intrinsic stress in sputtered thin films," Journal of Vacuum Science \& Technology A, vol. 9, no. 4, pp. 2431-2436, 1991.

[30] F. M. D’Heurle, "Aluminum films deposited by rf sputtering," Metallurgical and Materials Transactions B: Process Metallurgy and Materials Processing Science, vol. 1, no. 3, pp. 725-732, 1970.

[31] C. A. Davis, "A simple model for the formation of compressive stress in thin films by ion bombardment," Thin Solid Films, vol. 226, no. 1, pp. 30-34, 1993.

[32] M. Ohring, Materials Science of Thin Films: Deposition and Structure, Academic Press, San Diego, Calif, USA, 2nd edition, 2002. 

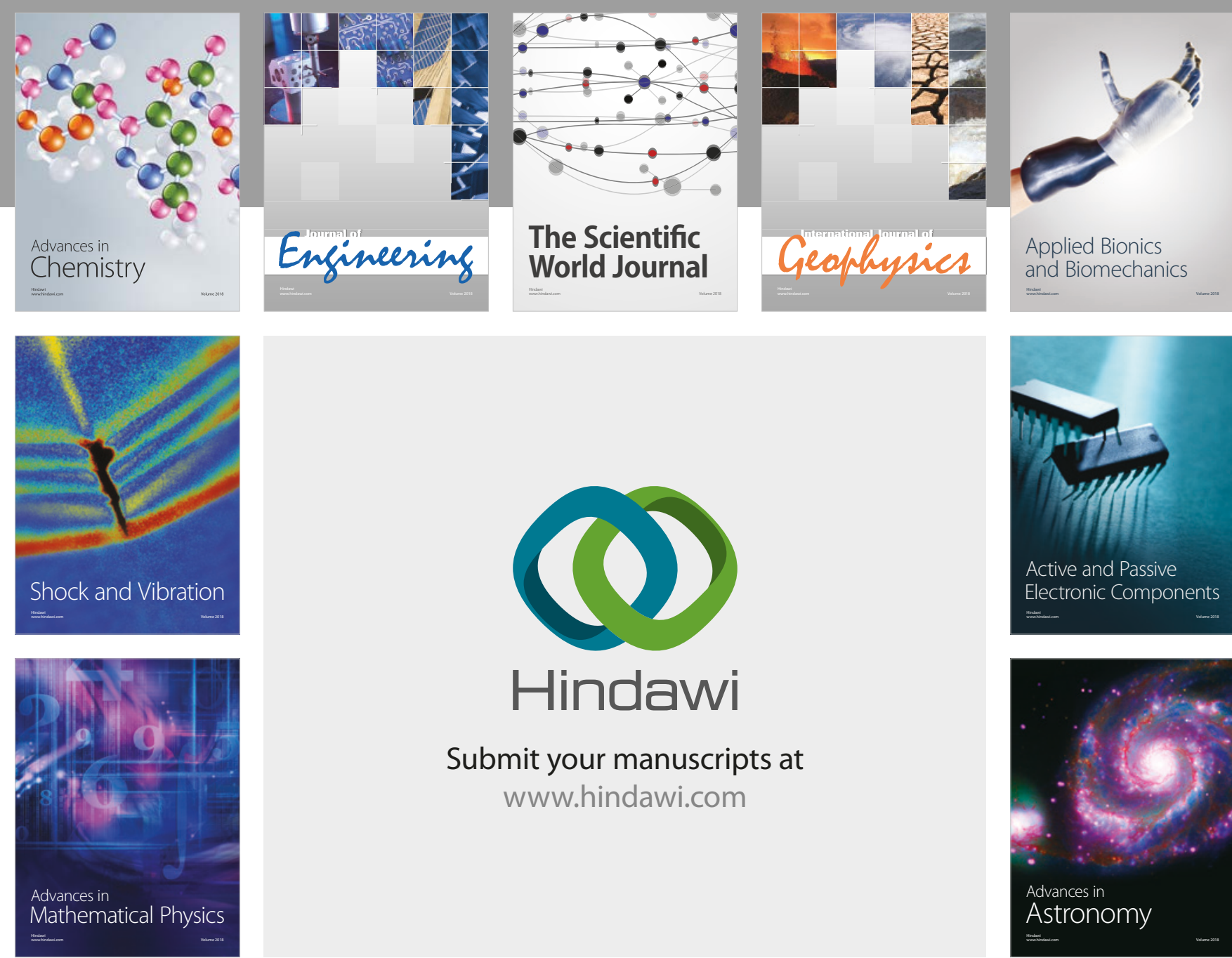

Submit your manuscripts at

www.hindawi.com

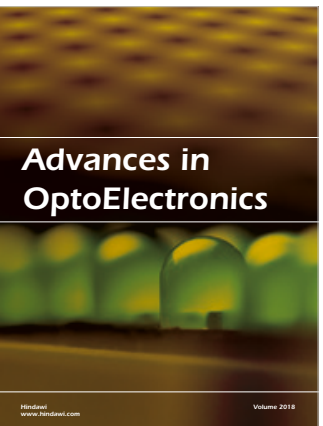

\section{Rotcting Machinery}
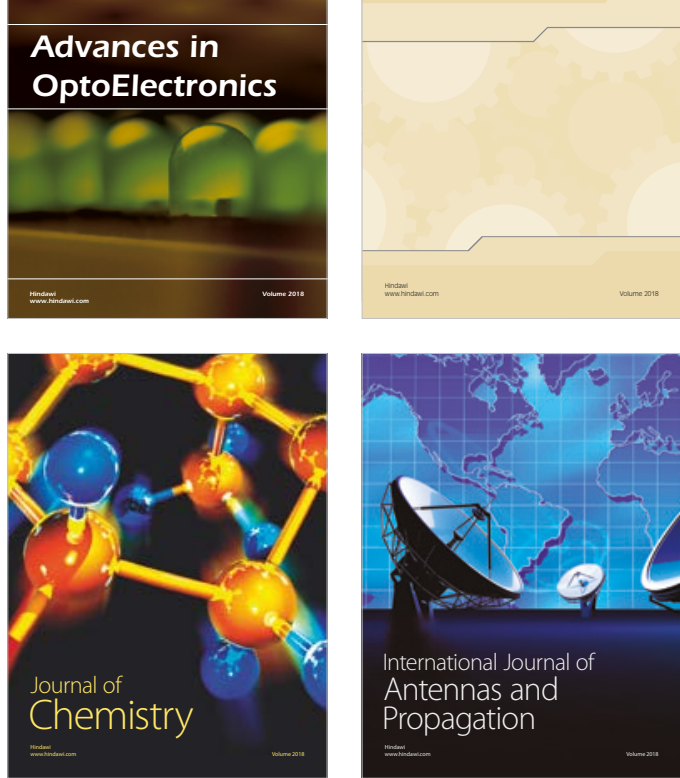

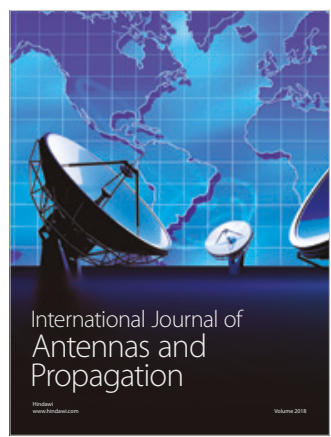

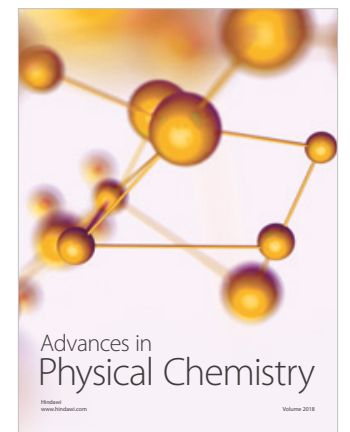

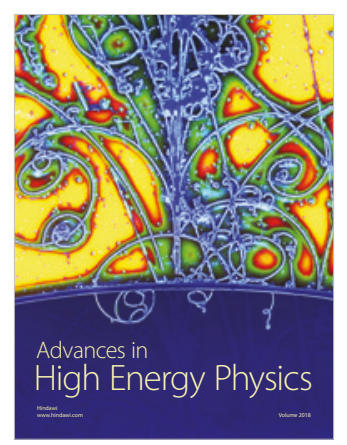

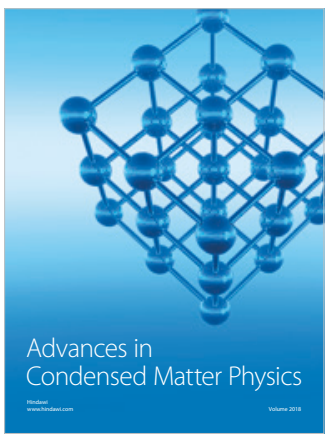

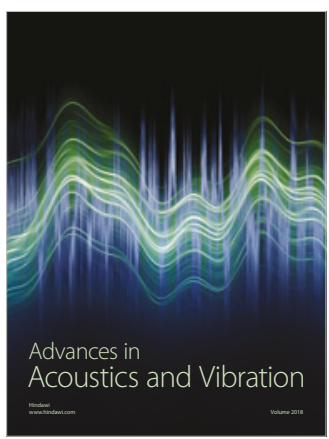

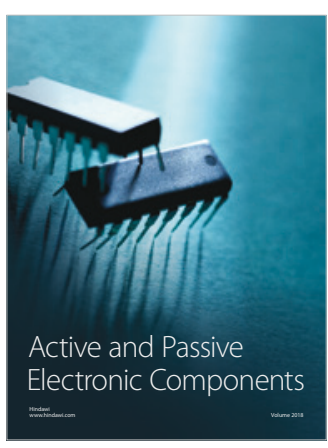
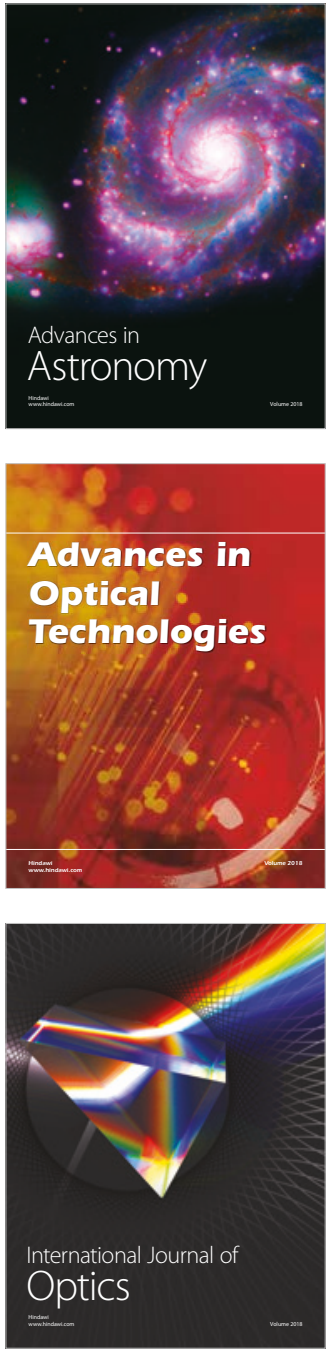